\title{
Estudo comparativo entre as anastomoses cervicais esofagogástrica término-terminal com e sem invaginação após esofagectomia para câncer
}

\section{Comparative study of end-to-end cervical esophagogastric anastomosis with or without invagination after esophagectomy for cancer}

\author{
Alexandre Cruz Henriques, TCBC-SP1; Aline Biral Zanon²; Carlos Alberto Godinho3; Lourdes Conceição Martins ${ }^{4}$;
} Roberto SAAd Junior, TCBC-SP5; Manlio Basilio Speranzini, ECBC-SP6; Jaques WaisberG, TCBC-SP7

\section{RE S U M O}

\begin{abstract}
Objetivo: Avaliar a incidência de fístula e estenose da anastomose esofagogástrica cervical com invaginação do coto esofágico no interior do estômago na esofagectomia para tratamento do carcinoma do esôfago. Métodos: Foram estudados dois grupos de doentes com carcinoma do esôfago torácico ou abdominal submetidos à esofagectomia subtotal e esofagogastroplastia. O grupo I (estudo) foi constituído por 29 doentes operados no período de 1998 a 2007, no qual foi realizada a anastomose esofagogástrica cervical com invaginação de segmento do coto esofágico no interior do estômago. O grupo II (controle) foi constituído por 36 doentes operados no período de 1989 a 1997 submetidos à anastomose esfagogástrica cervical término-terminal sem invaginação. Resultados: No grupo I, $3(10,3 \%)$ doentes apresentaram fístula da anastomose esofagogástrica com repercussão clínica mínima. No grupo ॥ observou-se fístula com franca saída de saliva em 11 (30,5\%) doentes. A freqüência de fístula nos doentes do grupo I foi significantemente menor $(p=0,04)$ do que nos do grupo II. No grupo I, estenose fibrótica da anastomose ocorreu em $7(24,1 \%)$ enfermos, ao passo que no grupo II $10(27,7 \%)$ evoluíram com estenose, não se constatando diferença significante $(p=0,72)$ entre esses grupos. Conclusão: No tratamento do carcinoma do esôfago, a esofagectomia com anastomose esofagogástrica cervical com invaginação do coto esofágico no interior do estômago determina menor ocorrência de fístula esofagogástrica quando comparado à anastomose sem invaginação. A incidência de estenose da anastomose esofagogástrica não diferiu em ambos os grupos.
\end{abstract}

Descritores: Neoplasias esofágicas. Esofagectomia. Fístula esofágica.

\section{INTRODUÇÃO}

O tratamento cirúrgico com intenção curativa do carcinoma do esôfago permanece um desafio ao cirurgião que lida com esta afecção ${ }^{1-8}$.

Trata-se de operação de grande porte com mortalidade que pode atingir $24 \%$ dos doentes e morbidade que pode chegar a $50 \%$ representada, entre outras, por complicações pulmonares, fístula cervical, estenose da anastomose, necrose da víscera transposta e mediastinite ${ }^{5,9-12}$

Dentre as várias complicações possíveis, a fístula da anastomose esofagogástrica permanece como um dos principais problemas das esofagectomias. Sua incidência varia de 0 a $50 \%$, porém a maioria dos autores relata incidência elevada ${ }^{4,6,13-18}$. Embora as fístulas habitualmente apresentem evolução clínica favorável, cerca de $2 \%$ delas podem evoluir de maneira catastrófica ${ }^{5,16}$. Quando a fístula não é a causa do óbito, ela colabora para piorar a qualidade de vida dos doentes, impede a realimentação precoce, exige cuidados locais trabalhosos e prolonga o tempo de internação. Além disso, dos doentes que desenvolvem fístula $30 \%$ a $50 \%$ evoluem para estenose ${ }^{15,19}$.

De maneira geral, a estenose ocorre em cinco a $46 \%$ dos casos operados e pode se manifestar até um ano após a operação. Além da fístula, outros fatores contribuem para a formação da estenose: insuficiência cardíaca, isquemia do fundo gástrico e realização de anastomose mecânica ${ }^{15,19,20}$

Diante da alta incidência da fístula esofagogástrica e da importante morbi-mortalidade por ela acarretada, várias manobras técnicas foram descritas para diminuir sua freqüência: proteção da anastomose com cola de fibrina ${ }^{21}$, anastomose em dois tempos ${ }^{22}$, rotação do fundo gástrico ${ }^{23}$, revascularização microcirúrgica da víscera

Trabalho realizado no Hospital de Ensino da Faculdade de Medicina do ABC, São Bernardo do Campo - São Paulo - BR.

1. Professor Assistente da Disciplina do Aparelho Digestivo da Faculdade de Medicina do ABC - São Bernardo do Campo - SP - BR. 2. Acadêmica do $4^{\circ}$ ano do curso de Medicina da Faculdade de Medicina do ABC - São Bernardo do Campo - SP-BR. 3. Professor Colaborador da Disciplina do Aparelho Digestivo da Faculdade de Medicina do ABC - São Bernardo do Campo - SP - BR. 4. Professor Assistente da Disciplina de Saúde Coletiva da Faculdade de Medicina do ABC - São Bernardo do Campo - SP-BR. 5. Professor Titular da Disciplina de Cirurgia Torácica da Faculdade de Ciências Médicas da Santa Casa de São Paulo - São Paulo - SP-BR. 6. Professor Titular da Disciplina de Cirurgia do Aparelho Digestivo da Faculdade de Medicina do ABC - São Bernardo do Campo - SP-BR. 7. Professor Assistente da Disciplina de Cirurgia de Aparelho Digestivo da Faculdade de Medicina do ABC - São Bernardo do Campo - SP-BR. 
transposta ${ }^{24}$, anastomose mecânica ${ }^{15}$, confecção laparoscópica do tubo gástrico cinco dias antes da esofagectomia ${ }^{18}$, preservação da arcada vascular do hilo esplênico ${ }^{25}$, administração de prostaglandina E1 ${ }^{26}$ e anastomose com invaginação ${ }^{27-29}$.

Kleinsasser et al. ${ }^{27}$ realizaram, em cães, anastomose esôfago-esofágica com invaginação, confeccionando segmento tubular de mucosa e submucosa com $0,5 \mathrm{~cm}$ de extensão no coto proximal e retirando segmento semelhante do coto distal, de tal maneira que ao invaginarem o segmento de mucosa e submucosa proximal no segmento distal, recompunham todas as estruturas da parede esofágica. Porém, os resultados com essa técnica não foram conclusivos.

Haight ${ }^{28}$ relatou correção de fístula esôfagotraqueal com sucesso em recém-nascidos utilizando a anastomose esôfago-esofágica por telescopagem de todas as camadas do coto cranial no caudal.

Nigro ${ }^{29}$ realizou, em cães, anastomose esôfagoesofágica com invaginação: confeccionava no coto proximal segmento de submucosa-mucosa de $2,0 \mathrm{~cm}$ de extensão que era invaginado no coto distal que permanecia com todas as suas paredes intactas. A sutura era realizada com pontos separados de prolene 3.0 aplicados somente nas túnicas adventícia e muscular dos cotos esofágicos. Esse autor concluiu que esta anastomose é segura e eficiente.

Szücs et al. ${ }^{30}$ descreveram 108 doentes submetidos à esofagectomia com anastomose esofagogástrica com telescopagem de coto esofágico de 10 a $15 \mathrm{~mm}$ de extensão no interior do estômago, dos quais $12(11,1 \%)$ enfermos apresentaram fístula da anastomose.

Diante deste panorama, tendo como experiência pessoal uma alta incidência de fístula esofagogástrica cervical no tratamento do carcinoma esofágico ${ }^{31}$, decidimos executar a anastomose esofagogástrica cervical com invaginação de segmento do coto esofágico no interior do estômago transposto para a região cervical e analisar a incidência de fístula e estenose da anastomose esofagogástrica cervical com invaginação.

\section{MÉTODOS}

O projeto de pesquisa foi aprovado pelo Comitê de Ética em Pesquisa da Faculdade de Medicina do ABC.

Foram estudados dois grupos de doentes com carcinoma do esôfago torácico ou abdominal submetidos à esofagectomia subtotal e esofagogastroplastia. O Grupo I (estudo) foi constituído por 29 doentes nos quais foi realizada a anastomose esofagogástrica com invaginação no período de 1998 a 2007. O grupo II (controle) foi constituído por 36 doentes nos quais foi realizada anastomose esofagogástrica término-terminal, sem invaginação no período de 1989 a 1997. Todos os pacientes de ambos os grupos foram operados pelo mesmo cirurgião.

Os critérios de inclusão dos doentes para a realização da esofagectomia e anastomose esofagogástrica cervical com invaginação foram: esofagograma sem des- vio anormal do eixo do órgão, lesões com extensão longitudinal de até $5,0 \mathrm{~cm}$, ausência de sinais de invasão da árvore respiratória na fibrobroncoscopia, ausência de sinais de irressecabilidade da lesão esofágica ou de disseminação neoplásica à distância na tomografia torácica e abdominal.

No Grupo I, 25 (86,2\%) doentes eram homens e quatro (13,8\%) mulheres, com média de idade de 60,2 anos (44 a 74 anos). A lesão localizava-se no terço médio do esôfago em 15 (51,8\%) doentes e no terço inferior em $14(48,2 \%)$.

O diagnóstico foi confirmado por endoscopia digestiva alta e biópsia. Vinte e três $(79,3 \%)$ doentes eram portadores de carcinoma espinocelular e seis $(20,7 \%)$ de adenocarcinoma.

O estadiamento foi realizado pelo exame físico, radiografia do tórax, esofagograma e tomografia torácica e abdominal. A fibrobroncoscopia pré-operatória foi realizada nos doentes com lesão situada no terço médio do esôfago. Para comparação entre os dois grupos de doentes, foi considerado estadiamento inicial os estádios I e II e estadiamento avançado os estádios III e IV da classificação $\mathrm{TNM}^{32}$.

Os doentes que não atendessem os critérios de inclusão ou que apresentassem estado clínico comprometido e/ou doença sistêmica grave de difícil compensação que contra-indicassem o procedimento anestésico e/ou operatório foram excluídos do estudo.

Todos os doentes foram submetidos à avaliação clínica pré-operatória: 13 (44,8\%) doentes exibiram desnutrição grave com perda ponderal superior a $20 \%$ do peso habitual; oito (22,5\%) doentes apresentavam hipertensão arterial sistêmica e quatro $(13,7 \%)$ tinham doença pulmonar obstrutiva crônica. Dezessete (58\%) doentes eram simultaneamente etilistas (uma ou mais doses de bebidas destiladas por dia) e tabagistas (dez ou mais cigarros por dia).

Na ausência de contra-indicação, a esofagectomia subtotal seguida de esofagogastroplastia foi executada. Em 19 (65,5\%) doentes a operação foi realizada por via abdominal e cervical (trans-hiatal) e em 10 $(34,5 \%)$ por via torácica, abdominal e cervical. Todas as operações foram realizadas em tempo cirúrgico único e com duas equipes, uma atuando na região abdominal e outra na região cervical. Linfadenectomia foi realizada no campo abdominal e mediastino inferior. Em todos os casos o estômago foi transposto à região cervical pelo mediastino posterior.

Qualquer que fosse a via de acesso, o esôfago foi dissecado e isolado de suas estruturas vizinhas nas regiões cervical, torácica e abdominal. Nos tumores distais, o esôfago foi seccionado na região cervical com o cuidado de preservar o coto proximal com comprimento suficiente para que $4,0 \mathrm{~cm}$ do órgão pudesse ser invaginado no interior do estômago, preservando margem de segurança igual ou maior a $5,0 \mathrm{~cm}$. O esôfago foi, então, tracionado para a região abdominal, e o estômago seccionado com grampeador linear seguindo uma linha que se iniciava num 
ponto na pequena curvatura aproximadamente 5,0 cm abaixo da transição esofagogástrica e que se estendia diagonalmente ao fundo gástrico, liberando a peça operatória.

Nas lesões do terço médio, para garantir margem de segurança, o estômago foi seccionado em primeiro lugar e a peça foi retirada pela região cervical, onde ela era minuciosamente examinada e o ponto de secção com margem segura era escolhido. Se não houvesse margem adequada, a anastomose com invaginação era abandonada, procedendo-se a anastomose término-terminal sem invaginação.

Na região escolhida para confecção da anastomose foi realizada miotomia transversal em toda a circunferência da víscera (Figura 1). Na borda proximal da miotomia foi anastomosado o coto esofágico com o estômago transposto para a região cervical. Inicialmente foi confeccionada a anastomose da parede posterior com pontos de polydioxanone 4.0; ao término desta, o segmento de 4,0cm de esôfago foi invaginado no interior do estômago e a seguir foi realizada a sutura da parede anterior da mesma maneira que da parede posterior (Figura 2). Piloroplastia extramucosa pela técnica de Weinberg foi realizada em todos os doentes. Sonda nasoenteral foi introduzida em todos os pacientes. A região cervical foi drenada com dreno laminar.

Dieta por via oral foi iniciada, habitualmente no $10^{\circ}$ pós-operatório, na ausência de sinais de fístula esofagocutânea. Na presença de fístula da anastomose esofagogástrica, cuidados locais foram instituídos, mantendo-se a dieta pela sonda nasoenteral. A dieta por via oral foi iniciada após o fechamento da fístula. Na alta hospitalar, os doentes foram encaminhados para acompanhamento ambulatorial, onde foi realizado o planejamento de tratamento adjuvante.

No Grupo II, os critérios de inclusão e de exclusão para a realização da esofagectomia foram os mesmos do Grupo I. O Grupo II foi constituído por 36 doentes com carcinoma do esôfago submetidos à esofagectomia subtotal e esofagogastroplastia com anastomose esofagogástrica término-terminal. Trinta e três $(91,6 \%)$ eram do sexo masculino e três $(8,4 \%)$ do feminino, com média de idade de 55.7 anos (38 a 75 anos). A lesão encontrava-se no terço médio do esôfago em 23 (63,8\%) doentes e no terço inferior em $13(36,2 \%)$.

O diagnóstico da neoplasia esofágica foi confirmado por endoscopia digestiva alta e biópsia. Vinte e nove doentes $(80,5 \%)$ eram portadores de carcinoma espinocelular e $7(19,5 \%)$ de adenocarcinoma.

A avaliação clínica pré-operatória e o estadiamento foram realizados de maneira semelhante ao Grupo I. Vinte e dois $(61,1 \%)$ doentes exibiram desnutrição grave com perda ponderal superior a $20 \%$ do peso habitual; três (8,3\%) apresentavam hipertensão arterial sistêmica, cinco $(13,8 \%)$ doença pulmonar obstrutiva crônica e quatro $(11,1 \%)$ diabete melito. Vinte e oito $(77,7 \%)$ doentes eram simultaneamente etilistas (uma ou mais doses de bebida destilada por dia) e tabagistas (vinte ou mais cigarros por dia).

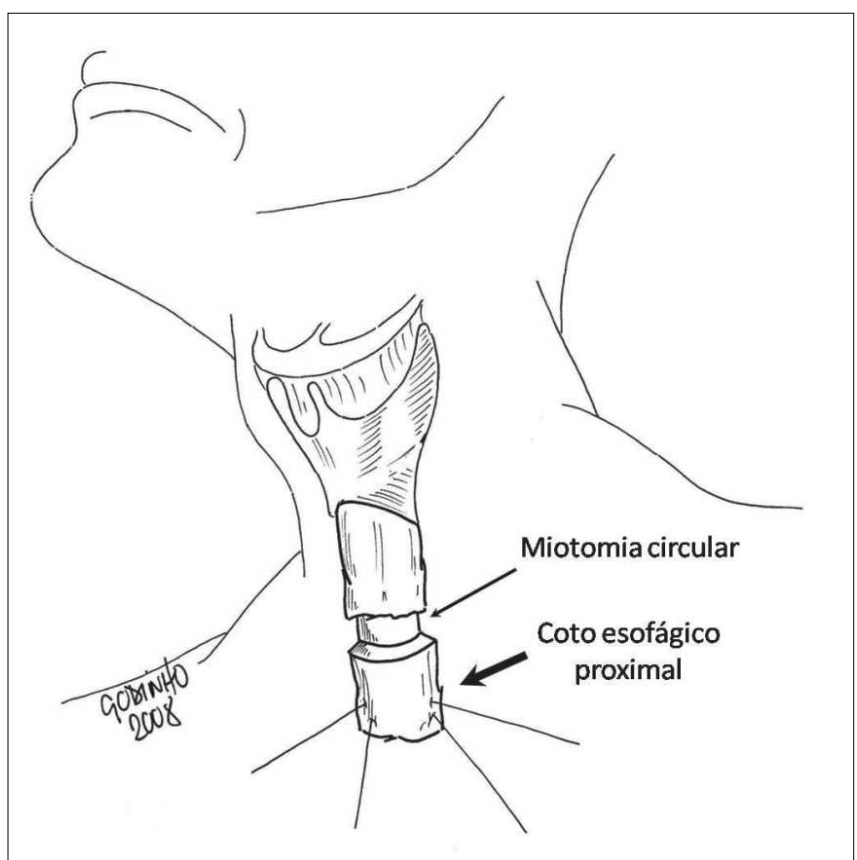

Figura 1 - Miotomia circular (seta fina) no coto esofágico criando segmento de $4,0 \mathrm{~cm}$ de extensão (seta larga) a ser invaginado no interior do estômago.

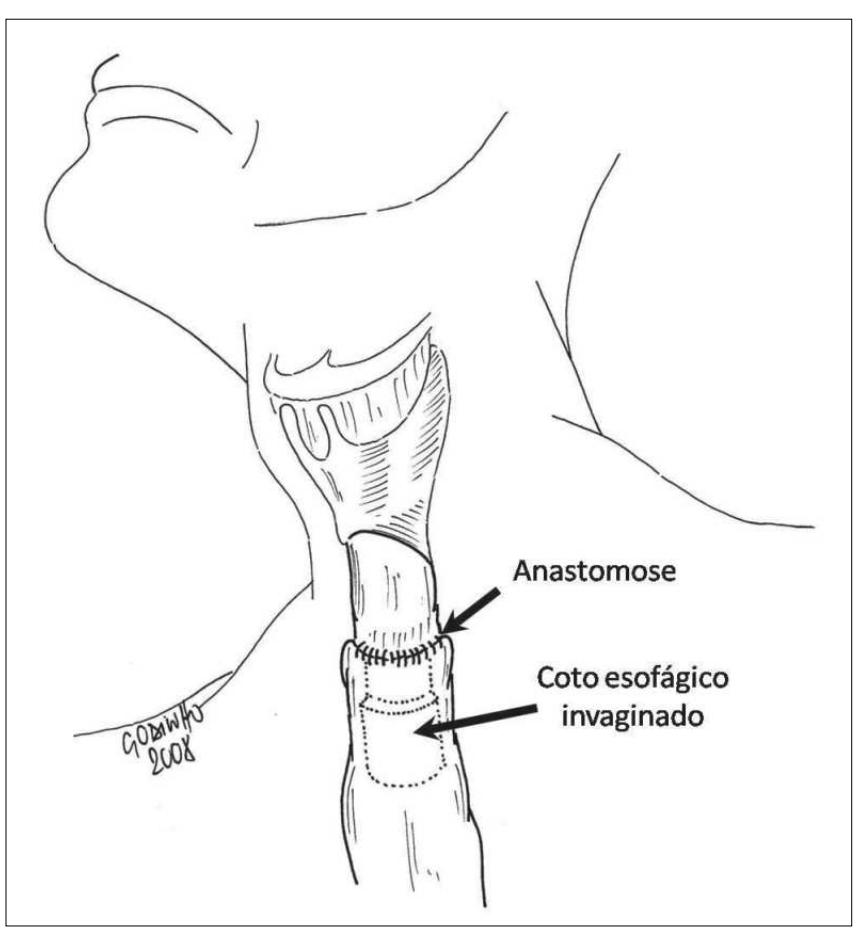

Figura 2 - Coto esofágico invaginado no interior do estômago: pontos aplicados na borda proximal da miotomia e na borda gástrica.

Da mesma forma, o preparo pré-operatório e a técnica operatória foram semelhantes nos dois grupos, exceto em dois aspectos: nos doentes do Grupo II, a anastomose esofagogástrica cervical era realizada de maneira término-terminal e neste grupo nenhum doente foi operado por via transtorácica. Os cuidados pós-operatórios foram os mesmos aplicados aos enfermos do Grupo I. 
Foram utilizados os seguintes modelos estatísticos: média aritmética e desvio padrão, e teste do "qui" quadrado. Em todos os testes, fixou-se em 0,05 ou $5 \%$ o nível para a rejeição da hipótese de nulidade (intervalo de confiança de 95\%) de acordo com os padrões correntes em estudos biológicos.

\section{RESULTADOS}

Não se observou diferenças significantes entre os dois grupos de doentes no tocante ao sexo $(p=0,37)$, tipo histopatológico $(0,57)$, localização da neoplasia $(p=0,23)$, grau de desnutrição $(p=0,14)$, hipertensão arterial essencial $(p=0,22)$, doença pulmonar obstrutiva crônica $(p=0,63)$, diabete melito $(p=0,08)$, etilismo e tabagismo $(p=0,82)$ e estadiamento inicial ou avançado da neoplasia $(p=0,28)$.

No Grupo I não houve óbito intra-operatório. Complicações pós-operatórias ocorreram em 21 (72,4\%) doentes desse grupo. Quatro (13,7\%) enfermos apresentaram complicações consideradas graves que culminaram com o óbito: dois $(6,9 \%)$ devido à broncopneumonia, um $(3,4 \%)$ em virtude de falência de múltiplos órgãos após reoperação para tratamento de colecistite aguda alitiásica e outro $(3,4 \%)$ em conseqüência a sepse por necrose isquêmica do tubo gástrico.

No Grupo I, três (10,3\%) doentes apresentaram fístula da anastomose esofagogástrica com débito mínimo de ar e saliva. Dois $(6,9 \%)$ deles apresentaram fístula no $7^{\circ}$ e no $11^{\circ}$ pós-operatório, com saída de mínima quantidade de ar e saliva pela incisão cervical e conseqüente formação de "bolha" durante os movimentos de deglutição; o fechamento espontâneo ocorreu, respectivamente, após dez e cinco dias. O terceiro doente (3,6\%) apresentou, no $13^{\circ}$ pós-operatório, derrame pleural seropurulento que foi drenado. O teste do azul de metileno demonstrou saída de mínima quantidade de corante pelo dreno pleural, demonstrando fístula esofagopleurocutânea de baixo débito e bloqueada. Não houve deterioração do estado geral e o doente recebeu alta no $23^{\circ}$ pós-operatório. Nenhum doente apresentou fístula da anastomose esofagogástrica com saída franca de saliva pela cervicotomia ou pelo dreno torácico.

No Grupo I, estenose fibrótica da anastomose ocorreu em sete $(24,1 \%)$ doentes; em seis deles, o seu aparecimento variou de 16 a 60 dias; em um doente, a estenose se manifestou 12 meses após a operação. Todos os doentes com estenose tiveram alívio da disfagia com a dilatação endoscópica da anastomose e o número de sessões variou de uma a sete (média de três sessões). Um $(3,6 \%)$ enfermo apresentou estenose por recidiva neoplásica na área de anastomose e necessitou de passagem de sonda enteral. As demais complicações foram tratadas clinicamente com sucesso: disfonia em $14(48,2 \%)$ doentes, broncopneumonia em quatro $(13,7 \%)$, atelectasia em dois $(6,8 \%)$, insuficiência renal em dois $(6,8 \%)$ e supuração da ferida operatória em um (3,4\%). A média do tempo de internação foi de 15,2 dias (13 a 35 dias).
O estadiamento clinicopatológico pela classificação TNM da UICC ${ }^{31}$ dos doentes submetidos à anastomose esofagogástrica com invaginação do coto esofágico foi: estádio I em dois $(6,8 \%)$ doentes, estádio lla em quatro(13,8\%), estádio IIb em quatro(13,8\%), estádio III em $15(51,7 \%)$ e estádio IV em quatro (13,8\%) enfermos. Foram classificados como estádio inicial $10(34,4 \%)$ doentes e em estádio avançado 19 (65,5\%) enfermos.

No Grupo Il também não houve óbito intra-operatório. Nesse grupo, complicações pós-operatórias ocorreram em 29 (80,5\%) doentes. Quatro (11,1\%) doentes apresentaram complicações graves (três casos de broncopneumonia e um caso de embolia pulmonar) que evoluíram para óbito.

No Grupo II, 11 (30,6\%) doentes apresentaram fístula da anastomose esofagogástrica que se manifestou entre o $5^{\circ}$ e $9^{\circ}$ pós-operatório. Em todos os casos ocorreu franca saída de saliva pela incisão cervical com fechamento espontâneo entre o $14^{\circ}$ e $30^{\circ}$ pós-operatório. Dez $(27,7 \%)$ doentes evoluíram com estenose da anastomose esofagogástrica e foram tratados com sucesso pela dilatação endoscópica. Ocorreram outras complicações tratadas clinicamente com sucesso: disfonia em nove (25\%) doentes, broncopneumonia em quatro $(11,1 \%)$, crise hipertensiva em um $(2,7 \%)$, insuficiência renal em dois ( 5,5\%) e insuficiência cardíaca em um $(2,7 \%)$ enfermo.

O estadiamento clinicopatológico pela classificação TNM da UICC ${ }^{32}$ dos doentes do Grupo II foi: estádio lla em cinco $(13,9 \%)$ doentes, estádio llb em quatro $(11,1 \%)$, estádio III em $23(63,8 \%)$ e estádio IV em quatro $(11,1 \%)$ enfermos. Foram classificados como estádio inicial nove (25,0\%) doentes e em estádio avançado 27 (74,9\%) enfermos.

Na comparação entre os dois grupos de doentes, verificou-se que o número de fístulas no Grupo II foi significantemente maior $(p=0,04)$ do que nos enfermos do grupo I (Tabela 1). No tocante ao número de estenoses, não se observou diferença significante $(p=0,72)$ entre os dois grupos de doentes (Tabela 2).

Nos doentes de ambos os grupos não se observou relação significante $(p>0,05)$ entre a ocorrência de fístula ou estenose com as seguintes variáveis: tipo histológico da neoplasia, localização no esôfago, desnutrição grave, hipertensão arterial sistêmica, doença pulmonar obstrutiva crônica, diabete melito, etilismo e tabagismo, via de acesso e estadiamento inicial ou avançado.

Tabela 1 - Incidência de fístula da anastomose esofagogástrica com invaginação (grupo l) ou sem invaginação (grupo II).

\begin{tabular}{lcllll}
\hline & Grupo I N & Grupo II N & P \\
\hline Com fístula & 3 & $(10,3 \%)$ & 11 & $(30,6 \%)$ & $0,04 *$ \\
Sem fístula & 26 & $(89,7 \%)$ & 25 & $(69,4 \%)$ & \\
Total de doentes & $29(100 \%)$ & $36(100 \%)$ & \\
\hline
\end{tabular}

$N=$ Número de doentes $\quad *$ = Significante. 
Tabela 2 - Incidência de estenose da anastomose esofagogástrica com invaginação (grupo I) ou sem invaginação (grupo II).

\begin{tabular}{|c|c|c|c|}
\hline & Grupo I N & Grupo II N & $\mathrm{P}$ \\
\hline Com estenose fibrótica & $7 \quad(24,1 \%)$ & $10 \quad(27,7 \%)$ & 0,72 (N.S.) \\
\hline Com estenose neoplásica & $1 \quad(3,6 \%)$ & _- & \\
\hline Sem estenose fibrótica & $22 \quad(72,3 \%)$ & $26 \quad(72,2 \%)$ & \\
\hline Total de doentes & $29(100 \%)$ & $36(100 \%)$ & \\
\hline
\end{tabular}

$N=$ Número de doentes $\quad$ N.S. = Não significante.

\section{DISCUSSÃO}

Sempre que possível, a esofagectomia é o tratamento de eleição para o carcinoma do esôfago ${ }^{3,8}$. Trata-se de operação de grande porte com índice de mortalidade operatória entre 0 a $24 \% \%^{5,9,12}$. No presente estudo, este índice foi de $13,7 \%$ nos doentes submetidos à técnica de invaginação da anastomose esofagogástrica e de 11,1\% nos enfermos com reconstrução do trânsito esofagogástrico sem invaginação anastomótica.

A morbidade da esofagectomia também é considerada elevada ${ }^{5,9,12}$. No presente estudo, a freqüência elevada de complicações pós-operatórias nos doentes do Grupo I $(72,4 \%)$ e do Grupo II $(80,5 \%)$ atesta a morbidade elevada que habitualmente acompanha as ressecções esofágicas para o tratamento do carcinoma. Dentre as complicações pós-operatórias, a fístula se destaca quer pela sua freqüência, quer pela gravidade, que pode ser devastadora quando determina mediastinite ${ }^{4,6,13-19}$.

A anastomose esofagogástrica com invaginação constitui modificação técnica realizada com intenção de diminuir a freqüência da fístula desta anastomose ${ }^{30}$. Optamos por invaginar segmento de $4 \mathrm{~cm}$, mais longo do que o preconizado por Szucs et al. ${ }^{30}$ e constituído de todas as camadas da parede do esôfago. Nossa intenção não era apenas recobrir internamente a zona da anastomose, mas também promover o deságüe de saliva numa região abaixo dela, tentando deixar a anastomose "exclusa" do trânsito. Para tal intento, era necessário invaginar segmento mais longo e constituído de todas as camadas da parede esofágica, de tal forma que o segmento invaginado permanecesse "armado", formando espécie de tubo no interior do estômago.

Para realizar a anastomose propriamente dita, escolhíamos região no coto esofágico proximal onde seria aplicada a sutura preservando $4,0 \mathrm{~cm}$ de esôfago para ser invaginado no estômago. Neste ponto era realizada miotomia transversal em toda a circunferência do esôfago e na borda proximal desta miotomia, era executada a sutura com a camada seromuscular do estômago. A intenção desta miotomia era criar uma borda cruenta na camada muscular do esôfago para ser suturada com a camada seromuscular do estômago e também alongar o tubo esofágico que seria invaginado no estômago.

O ponto de secção do esôfago deve ser escoIhido sem negligenciar a margem de segurança, pois o carcinoma do esôfago apresenta disseminação intramural até regiões distantes da lesão principal ${ }^{8,33}$. Akiyama ${ }^{8}$ e Roth et al. ${ }^{33}$ preconizam margem de $5,0 \mathrm{~cm}$ de esôfago sem neoplasia para a ressecção segura, exceção feita para lesões no esôfago cervical e bem localizadas, quando margens cirúrgicas livres menores são toleradas. Para realizar a anastomose esofagogástrica cervical com invaginação é preciso conservar $4,0 \mathrm{~cm}$ a mais de esôfago proximal quando comparado ao nível de ressecção para a anastomose sem invaginação. Quando a lesão está situada no esôfago abdominal, a margem de segurança está garantida. Entretanto, quando a lesão localiza-se no esôfago cervical, a anastomose esofagogástrica com invaginação está contra-indicada e, muitas vezes, a própria esofagectomia. Quando o tumor é do esôfago torácico, a margem de segurança deve ser rigorosamente avaliada. Para obter-se margem cirúrgica livre pode ser utilizada a manobra preconizada por Akiyama ${ }^{8}$ que consiste em seccionar o estômago e tracionar toda a peça para a região cervical, onde poderá ser minuciosamente examinada e o ponto de secção com margem segura é escolhido. Se não houver margem adequada, a invaginação deve ser abandonada.

Julgamos que o fato desta técnica conservar $4,0 \mathrm{~cm}$ a mais de esôfago proximal não abole a radicalidade da operação. A esofagectomia à Ivor Lewis realizada por diversos autores ${ }^{34-36}$ utiliza como via de acesso, a laparotomia mediana e a toracotomia direita. Com essa técnica, a anastomose esofagogástrica é executada no ápice do tórax e, apesar de conservar $5,0 \mathrm{~cm}$ a mais de esôfago quando comparada com a anastomose cervical, esta operação é considerada radical ${ }^{10}$. Para a construção da anastomose esofagogástrica cervical com invaginação, o esôfago remanescente não é maior do que aquele deixado quando se executa a anastomose no ápice do tórax. Walther et al. ${ }^{6}$, em estudo prospectivo randomizado, compararam a anastomose esofagogástrica cervical com a anastomose esofagogástrica intra-torácica. Concluíram que a retirada de $5,0 \mathrm{~cm}$ a mais de esôfago para realização da anastomose no pescoço não alterou o índice de sobrevivência de cinco anos. Portanto, consideramos que, preservados os cuidados citados com a margem de segurança, a anastomose esofagogástrica com invaginação não fere os princípios da radicalidade oncológica. Na presente casuística, um doente do Grupo I com neoplasia do esôfago abdominal apresentou, no 
pós-operatório, estenose neoplásica na zona de anastomose. O exame anatomopatológico da peça operatória mostrou margens cirúrgicas livres, mas considerou-se que a recidiva na área da anastomose deveu-se à presença de infiltração neoplásica em algum ponto da margem distal não submetida ao exame microscópico, não guardando relação com a técnica da anastomose com invaginação.

O diagnóstico de fístula da anastomose esofagogástrica foi realizado exclusivamente em critérios clínicos, uma vez que o estudo radiológico com contraste hidrossolúvel apresenta baixa sensibilidade e grande número de falsos negativos ${ }^{37}$. Nenhum caso operado por esofagectomia e anastomose esofagogástrica cervical com invaginação apresentou fístula com saída franca de saliva pela incisão cervical. Comparando com os resultados descritos na literatura ${ }^{4,6,13-18}$ que apontam incidência de fístulas entre 0 a 50\%, julgamos que a anastomose esofagogástrica cervical com invaginação apresentou incidência baixa de fístula $(10,3 \%)$, e apenas um $(3,4 \%)$ caso apresentou repercussão clínica relevante. Por outro lado, no Grupo II, onde os doentes foram submetidos à anastomose esofagogástrica cervical com anastomose terminoterminal, $11(30,5 \%)$ doentes apresentaram fístula da anastomose esofagogástrica, todos com saída profusa de saliva pela incisão cervical, o que desencadeou a necessidade de cuidados locais, diminuindo a qualidade de vida do doente. Nesta série de doentes, a aplicação da técnica da invaginação na realização da anastomose esofagogástrica mostrou redução significante da incidência de fístula quando comparada com a técnica sem invaginação: 10,3\% e $30,6 \%$, respectivamente.

A anastomose esofagogástrica com invaginação possivelmente não modifica os fatores que predispõem a formação da fístula. Provavelmente, pontos de deiscência podem ocorrer na linha de sutura com a mesma freqüência quando se utiliza técnica de anastomose sem invaginação. No entanto, como a saliva deságua numa região abaixo da anastomose, estes pontos de deiscência sofrem rápida regeneração. Por outro lado, nos doentes com anastomose sem invaginação, a saliva deságua diretamente no local da deiscência, provocando inflamação e infecção local, dificultando os processos de cicatrização da linha de sutura e ampliando a área de deiscência.

Acreditamos que, em virtude deste mecanismo, os três casos de fístula da anastomose esofagogástrica observadas no grupo I apresentaram débito diminuto, mesmo no doente cuja fístula dirigiu seu trajeto para o espaço pleural. Julgamos que o resultado representado pela fístula com repercussão clínica mínima pode constituir vantagem da anastomose esofagogástrica com invaginação quando comparada com a anastomose sem invaginação.

A estenose da anastomose esofagogástrica apresenta incidência de 5 a $45 \%{ }^{19}$ e, via de regra, é considerada complicação não grave. Aproximadamente 50\% dos enfermos com fístula anastomótica evoluem para estenose $\mathrm{e}^{5,19,20}$. Felizmente, a maioria dos doentes obtém a permeação do lume esofágico com dilatação endoscópica da área estenosada ${ }^{19,20}$. Na presente casuística, sete $(24,1 \%)$ doentes do Grupo I e 10 (27,7\%) do Grupo II desenvolveram estenose fibrótica da anastomose, índices dentro dos valores apontados por outros autores que variaram de 5 a $46 \%^{15,19,20}$. Nos doentes submetidos à anastomose esofagogástrica sem invaginação, a incidência de estenose não foi significantemente diferente da dos enfermos com anastomose esofagogástrica com invaginação. Julgamos que este resultado ocorreu, possivelmente, devido ao fato de que a anastomose esofagogástrica com invaginação não modifica os fatores predisponentes para a formação da fístula, especialmente a isquemia da porção proximal da gastroplastia e, dessa maneira, não evita que pontos de deiscência ocorram na linha de sutura com a mesma incidência da anastomose sem invaginação. Porém a presença da fístula nem sempre foi identificada em bases clínicas, possivelmente devido ao fato da saliva desaguar abaixo do ponto de deiscência. Tais eventos podem desencadear reação fibrótica de cicatrização e conseqüente formação de estenose da anastomose.

No presente estudo, a ocorrência de fístula e/ou estenose nos doentes de ambos os grupos não se relacionou significantemente com variáveis morfológicas e clínicas. Esse resultado sugere que o aspecto técnico da confecção da anastomose esofagogástrica com invaginação pode ter sido fator contribuitivo importante para a incidência significantemente menor de fístula com repercussão clínica nos doentes operados com essa técnica.

As demais complicações encontradas no presente estudo foram inerentes ao tipo de operação empregada, fato também observado por outros autores ${ }^{5,9,10-12}$ e provavelmente não guardam relação direta com a anastomose esofagogástrica com invaginação.

Concluímos que a realização da anastomose esofagogástrica cervical com invaginação de segmento do coto do esôfago no interior do estômago na esofagectomia subtotal e gastroplastia nos doentes com carcinoma do esôfago torácico e abdominal está associada à menor incidência de fístula esofagogástrica, e incidência semelhante de estenose quando comparada à anastomose sem invaginação. Além disso, observamos que a repercussão clínica da fístula da anastomose esofagogástrica nos doentes em que foi realizada a técnica com invaginação é menor quando comparada com a anastomose sem invaginação.

A elaboração de estudos prospectivos randomizados e controlados para realização da anastomose esofagogástrica com invaginação de segmento do coto esofágico proximal no interior do estômago transposto à região cervical possui a potencialidade de confirmar os resultados obtidos na presente casuística. Dessa maneira, poderemos oferecer uma técnica mais segura para a confecção da anastomose esofagogástrica e melhorar a qualidade de vida dos desafortunados doentes com carcinoma do esôfago torácico e abdominal. 
Objective: To assess the incidence of fistula and stenosis of cervical esophagogastric anastomosis with invagination of the esophageal stump into the gastric tube in esophagectomy for esophagus cancer. Methods: Two groups of patients with thoracic and abdominal esophagus cancer undergoing esophagectomy and esophagogastroplasty were studied. Group I comprised 29 patients who underwent cervical esophagogastric anastomosis with invagination of the proximal esophageal stump segment within the stomach, in the period of 1998 to 2007 while Group II was composed of 36 patients submitted to end-to-end cervical esophagogastric anastomosis without invagination during the period of 1989 to 1997. Results: In Group I, esophagogastric anastomosis by invagination presented fistula with mild clinical implications in 3 (10.3\%) patients, whereas in Group II, fistulas with heavy saliva leaks were observed in 11 (30.5\%) patients. The frequency of fistulas was significantly lower in Group I patients $(p=0.04)$ than in Group II. In Group I, fibrotic stenosis of anastomoses occurred in 7 (24.1\%) subjects, and 10 patients (27.7\%) in Group II evolved with stenosis, while no significant difference $(p=0.72)$ was found between the two groups. Conclusion: In esophagectomy for esophagus cancer, cervical esophagogastric anastomosis with invagination presented a lower rate of esophagogastric fistula versus anastomosis without invagination. Stenosis rates in esophagogastric anastomosis proved similar in both approach with or without invagination.

Key words: Esophageal neoplasm. Esophagectomy. Esophageal fistula.

\section{REFERENCIAS}

1. Gockel I, Heckhoff S, Messow CM, Kneist W, Junginger T. Transhiatal and transthoracic resection in adenocarcinoma of the esophagus: does the operative approach have an influence on the long-term prognosis? World J Surg Oncol. 2005; 3(40):104-8.

2. Johansson J, DeMeester TR, Hagen JA, DeMeester SR, Peters JH, Oberg S, Bremner CG. En bloc vs transhiatal esophagectomy for stage T3N1, adenocarcinoma of the distal esophagus. Arch Surg. 2004; 139(6):627-31.

3. Korst RJ. Surgical resection of esophageal carcinoma: speaking the language. J Gastroenterol. 2005; 11(15):2211-2.

4. van Lanschot JJ, van Blankenstein M, Oei HY, Tilanus HW. Randomized comparison of prevertebral and retroesternal gastric tube reconstruction after resection of oesophageal carcinoma. $\mathrm{Br}$ J Surg. 1999; 86(1):102-8.

5. Rentz J, Bull D, Harpole D, Bailey S, Neumayer L, Pappas T et al. Transthoracic versus transhiatal esophagectomy: a prospective study of 945 pacients. J Thorac Cardiovasc Surg. 2003; 125(5):111420.

6. Walther B, Johansson J, Johnsson F, Von Holstein CS, Zilling T. Cervical or thoracic anastomosis after esophageal resection and gastric tube reconstruction: a prospective randomized trial comparing sutured neck anastomosis with stapled intrathoracic anastomosis. Ann Surg. 2003; 238(6): 803-12.

7. Siewert JR, Ott K. Are squamous and adenocarcinomas of the esophagus the same disease? Semin Radiat Oncol. 2007; 17(1):3844.

8. Akiyama H. Surgery for cancer of the esophagus. Baltimore: Willians \& Wilkins; 1990

9. Dimick JB, Goodney PP, Orringer MB. Birkmeyer JD. Specialty training and mortality after esophageal cancer resection. Ann Thorac Surg. 2005; 80(1):282-6.

10. Cassivi SD. Leaks, strictures, and necrosis: a review of anastomotic complications following esophagectomy. Semin in Thorac and Cardiovasc Surg. 2004; 16(2):124-32.

11. Goan YG, Chang HC, Hsu HK, Chon YP. An audit of surgical outcomes of esophageal squamous cell carcinoma. Eur J Cardiothorac Surg. 2007; 31(3): 536-44.

12. Dowson HM, Strauss D, Ng R, Mason R. The acute management and surgical reconstruction following failed esophagectomy in malignant disease of the esophagus. Dis Esophagus. 2007; 20(2):135-40

13. Horstmann O, Verreet PR, Becker H. Ohmann C, Röher HD. Transhiatal oesophagectomy compared with transthoracic resection and systematic linphadenectomy for the treatment of oesophageal cancer. Eur J Surg. 1995; 161(8):557-67.

14. Chu KM, Law SYK, Fok M, Wong J. A prospective randomized comparison of transhiatal and transthoracic resection for lowerthird esophageal carcinoma. Am J Surg. 1997; 174(3):320-4.
15. Orringer MB. Marshall B, lannettoni MD. Eliminating the cervical esophago-gastric anastomotic leak with a side-to-side stapled anastomosis. J Thorac Cardiovasc Surg. 2000; 119(2):277-88.

16. Korst RJ, Port JL, Lu PC, Altorki NK. Intrathoracic manifestation of cervical anastomotic leaks after transthoracic esophagectomy for carcinoma. Ann Thorac Surg. 2005; 80(4):1185-90.

17. Tinoco RC, Tinoco AC, El-Kadre LJ, Rios RA, Sueth DM, Pena FM. Esofagectomia laparoscópica transhiatal: resultados imediatos. Arq Gastroenterol. 2007; 44(2):141-4

18. Hölscher $A H$, Schneider PM, Gutschow C, Schöder W. Laparoscopic isquemia conditioning of the stomach for esophageal replacement. Ann Surg. 2007; 245(2):141-6.

19. Honkoop P, Siersema PD, Tilanus HW, Stassen LPS, Hop WCJ, Van Blankenstein M. Benign anastomotic strictures after transhiatal esophagectomy and cervical esophagogastrostomy: risk factors and management. J Thorac Cardiovasc Surg. 1996; 111(6):141-8.

20. Dewar L, Gelfand G, Finley RJ, Evans K, Inculet R, Nelems B. Factors affecting cervical anastomotic leak and stricture formation following esophagogastrectomy and gastric tube interposition. Am J Surg. 1992; 163 (5):484-9.

21. Thorson GIC, Perez-Brett R, Lillie DB, Ambrus JL, Takita H. The role of the tissue adhesive fibrin seal in esophageal anastomosis. J Surg Oncol. 1983; 24 (3):221-3.

22. Gurski RR, Schirmer CC, Toneto Jr JE, Rosa R, Toniazzo GP, Fasolo $\mathrm{GR}$, Brentano L. Delayed cervical anastomosis of the esophagus for esophageal carcinoma. Int Surg. 1997; 82(1):56-9.

23. Schilling M, Redaelli $C$, Zbären $P$, Baer HU, Seiler $C$, Friess $H$, Büchler MW. First clinical experience with fundus rotation gastroplasty as a substitute for the oesophagus. Br J Surg. 1997; 84(1):126-8.

24. Yoshimi F, Asato $Y$, Ibeda S, Okamoto K, Komunv $Y$, Imura J, Itabashi $M$. Using the supercharge technique to addionally revasularize the gastric tube after a subtotal esophagectomy for esophagear cancer. Am J Surg. 2006; 191(2):284-7.

25. Ueo H, Abe R. Takenchi H, Aninaga S, Akiyoshi T. A reliable operative procedure for preparing a sufficiently nourished gastric tube for esophageal reconstruction. Am J Surg. 1992; 165(2):273-6.

26. Matsuzaki Y, Edagawa M. Maeda M. Nakamura K, Onitsuka T. Beneficial effect of prostaglandin $E_{1}$ on blood flow to the gastric tube after esophagectomy. Ann Thorac Surg. 1999; 67(4):908-10.

27. Kleinsasser LJ, Cramer I, Warshaw H. Anastomosis of the cervical esophagus: experimental evaluation of peritoneal grafts. Surgery. 1950; 28(2):438-42.

28. Haight $C$. Congenital atresia of the esophagus with tracheoesophageal fistula. Ann Surg. 1944; 120(4):625-55.

29. Nigro AJ. Anastomose esôfago-esofágica cervical com invaginação: estudo experimental em cães. Acta Cir Bras. 1996; 11(2):63-75

30. Szücs G, Tóth K, Kiss I. Telescopic esophageal anastomosis: operative tecnique, clinical experiences. Dis Esophagus. 2003; 16(4):315-22.

31. Henriques AC, Pezollo S, Faure MG, da Luz LT, Godinho CA, Speranzini $\mathrm{MB}$. Gastro-esophageal isoperistaltic bypass as paliative treatment of the irresectable cancer. Rev Col Bras Cir. 2001; 28(6):408-13. 
32. Rice, TW. Diagnosis and staging of esophageal cancer. In Pearson's thoracic \& esophageal surgery. $3^{\text {rd }}$ ed. Oxford: Churchill Livingstone; 2008. p. 454-63.

33. Roth JA, Putmam JB. Surgery for cancer of the esophagus. Semin Oncol. 1994; 21(4):453-61.

34. Griffin SM, Shaw IH, Dresner SM. Early complications after Ivor Lewis subtotal esophagectomy with two field lumphadenectomy: risk factors and management. J Am Coll Surg. 2002; 194(3):28597.

35. Stilidi I, Davydov M, Bokhyan V. Subtotal esophagectomy with extended 2-field linphnode dissection for thoracic esophageal cancer. Eur J Cardiothorac Surg. 2003; 23(3):415-20.

36. Chung SCS, Stuart RC, Li AKC. Surgical therapy for squamous-cell carcinoma of the oesophagus. Lancet. 1994; 343(8):521-3.

37. Tirnacksig MB, Deschamps C, Allen MS, Johnson DC, Pairolero PC. Effectiviness of screening aqueous contrast swallow in detecting clinically significant anastomotic leaks after esophagectomy. Eur Surg Res. 2005; 37(2):123-8.
Recebido em 10/12/2008

Aceito para publicação em 16/02/2009

Conflito de interesse: nenhum

Fonte de financiamento: nenhuma

\section{Como citar este artigo:}

Henriques AC, Zanon AB, Godinho CA, Martins LC, Júnior R, Speranzini $M B$, Waisberg J. Estudo comparativo entre as anastomoses cervicais esofagogástricas término-terminal com e sem invaginação após esofagectomia para câncer. Rev Col Bras Cir. [periódico na Internet] 2009; 36(5). Disponível em URL: http://www.scielo.br/rcbc

\section{Endereço para correspondência:}

Alexandre Cruz Henriques

E-mail: achenriques@uol.com.br 